\title{
Construction of Ethylene Gas Detection System Using Neural Networks
}

\author{
Tatsuyuki Wada*, Sigeru Omatu \\ Dept. of Information and Communication Engineering, Osaka Institute of Technology \\ 5-16-1, Omiya, Asahi-ku, Osaka, 535-8585, Japan \\ m1m14328@st.oit.ac.jp,omatu@rsh.oit.ac.jp
}

\begin{abstract}
In this paper, we show the results on the ethylene gas detection. The ethylene gas is generated as one of the by-products of the breathing in many plants. It may become harmful to the freshness when we keep on having collected around a ripe product excessively. Ethylene gas accelerates a process to mature and caused self-acceleration of the deterioration. The main promotion of ethylene is extremely a small amount, but gas chromatography and mass spectrometry are used as the measurement devices which can separate gases. The components are expensive and need much experience for operation.

We use a neural network based on the features that can be obtained from measurement data assuming the real environment such as steam, alcohol, and ethylene. Using a small odor measurement system that created using the mass sensor of the inexpensive crystal oscillator can be detected selectively to the ethylene gas. We consider the technique to implemente and configure the system to show the processing result.
\end{abstract}

\section{Introduction}

In recent years, biological olfactory principle obviously becomes increasing sensor selectively which can detects odor ${ }^{(1)(2)(3)}$ of thin concentration by advances in molecular biology. In this paper, we propose a method for the ethylene gas detection. The odor sensor is a quartz oscillator; the price is cheaper than Metal Oxide Gas sensor. The sensor has discrimination ability in accordance and the purpose by selecting the type of the organic film to be coated on the electrode. ${ }^{(4)}$

We focused on configuring the ethylene gas detection system using these sensors.

Ethylene gas accelerates a process to mature and causes self-acceleration of the deterioration ${ }^{(5)}$. The maturity promotion action of ethylene is extremely a small amount, but gas chromatography and mass spectrometry are used as the measurement device.
These separate gas, and the technique to analyze the composition is expensive.

Therefore, we propose a low-cost and small system. We use a neural network ${ }^{(6)(7)}$ based on the features that can be obtained from measurement data assuming the real environment such as steam, alcohol, and ethylene. We created the small odor measurement system using the mass sensor of the inexpensive crystal oscillator. It can detect the ethylene of thin density. Finally, experimental result are shown to see the effectiveness

\section{Principle of $\mathrm{QCM}$ sensors}

The QCM has been well-known to provide very sensitive mass-measuring devices in nanogram levels. Synthetic polymer-coated QCMs have been studied as sensors for various gas works as a chemical sensor. The QCM sensors are made by covering the surface with several kinds of a thin membrane with about $1 \mathrm{~nm}$, as shown in Fig. 1. The QCM sensor is integrated into a resonance circuit. If the film absorbs the odor molecules, the oscillation frequency is reduced since the mass of the vibrator is heavier.

Therefore, the frequency of the QCM will change according to the deviation of the weight due to the

adsorbed odor molecular (odorant). In this paper, we have used the 7 sensors. Membrane materials each of sensors are shown in Table 1. The basic approach used here is a sol-gel method. The process is a wet-chemical technique used for the fabrication of both glassy and ceramic materials. The manufacturing of a film was done by the following procedure.

MTMS (1): Trimethylsilane, ethanol, water and nitric acid. (2) Once stirred, 2-ethyl acrylate (PFOEA) was added to a solution of 1.Here, HTMS: $\mathrm{CH} 3 \mathrm{Si}(\mathrm{OCH} 3) 3$ and PFOEA: F(CF2)8CH2CH2$\mathrm{OCOCH}=\mathrm{CH} 2$

Table 1 Chemical materials used us the membrane 
*F3=C4H12O3Si $(0.5 \mathrm{~g}), \mathrm{C} 16 \mathrm{H} 19 \mathrm{~F} 17 \mathrm{O} 3 \mathrm{Si}(0.015 \mathrm{~g}), 3$ $0 \% \mathrm{HNO} 3(10 \mu \mathrm{L}), \mathrm{H} 2 \mathrm{O}(0.30 \mathrm{ml})$, etanol $(3.0 \mathrm{~m})$

* F6 = C4H12O3Si $(0.5 \mathrm{~g}), \mathrm{C} 16 \mathrm{H} 19 \mathrm{~F} 17 \mathrm{O} 3 \mathrm{Si}(0.030 \mathrm{~g})$, $30 \% \mathrm{HNO} 3(10 \mu \mathrm{L}), \mathrm{H} 2 \mathrm{O}(0.15 \mathrm{ml})$, etanol $(3.0 \mathrm{ml})$

\section{Sensor number Materials of mebrane}

\begin{tabular}{ll}
\hline sensor1 & F3 \\
sensor2 & F3 \\
sensor3 & F3 \\
sensor4 & F6 \\
sensor5 & F6 \\
sensor6 & F6 \\
sensor7 & F6
\end{tabular}
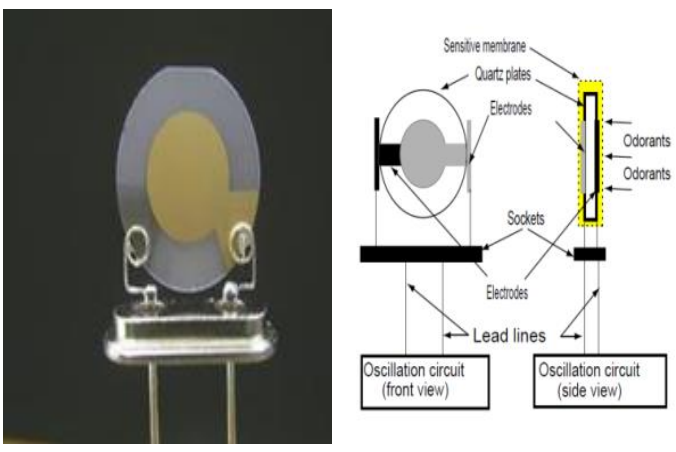

Fig. 1 Principle of QCM sensor.

\section{Odor Sensing Hardware System}

Generally, this type of system, for example an air purifier, a breathalyzer, etc., is designed to detect some specific odor. Each of the QCM membranes has its own characteristics in response to different odors. When combining many QCM sensors together, the ability to detect the odor is increased. Measurement circuit utilizes the principle of the QCM sensor, frequency-change recorded as output of the sensor. In this paper, we measured the odor using a measuring device arranged QCM sensor array (Fig.2). Fig.2 (a) shows white tubular that can hold a QCM sensor, and the measures BOX can react in gas flowing into the tube. In this research, we are measuring circuit using a QCM sensor of $20 \mathrm{MHz}$ by use of a circuit for outputting a difference between the oscillator $(20 \mathrm{MHz})$ in the frequency and the measurement circuit of the odor sensor, as shown in Fig. 2 (b).

Odor sensing systems, shown in Fig. 3, were developed based on the concept of the human olfactory system. First, the system allows the mix gas flow to the odor coming from the mixing device to the sampling box. Then the reaction of the QCM sensor is accumulated into frequency counter as data. In addition, the gas is exhausted from the sampling box. The PC converts the data stored in the frequency counter into a text file. Besides, If the measurement object is a liquid then using Permeater. This machine can continuously generate standard gas of many kinds (inorganic and organic gas) for a long time. The process takes place in the standard gas generator, which generates a continuous standard of trace gas concentration.

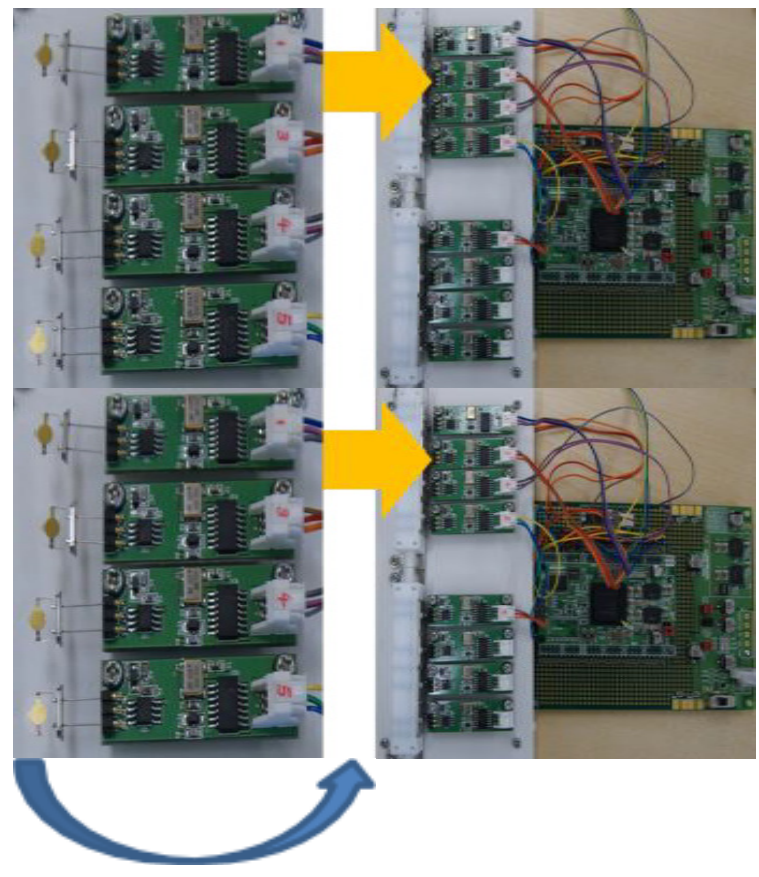

(a)circuit(QCM sensor are place inside the sampling tube)

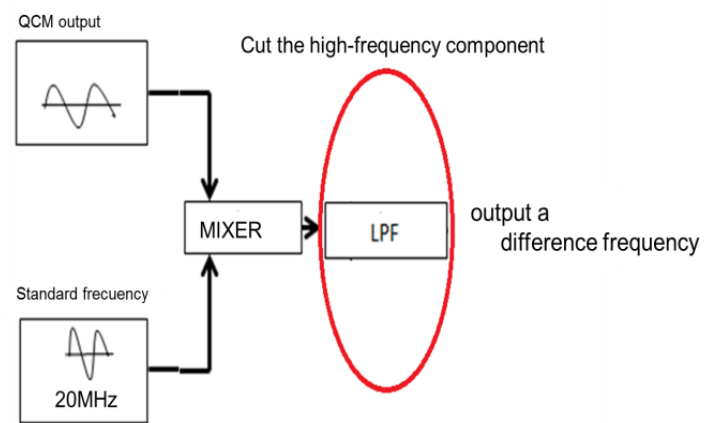

(b) Principle of odor measurement circuit

Fig. 2 Measurement circuit. 


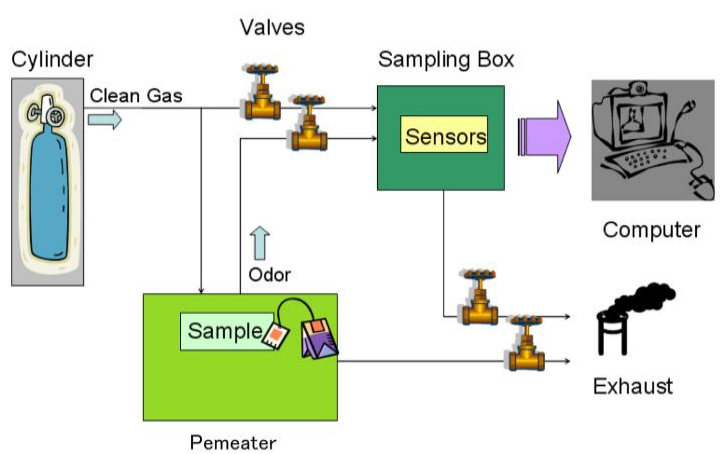

(a) When the measurements object is a liquid

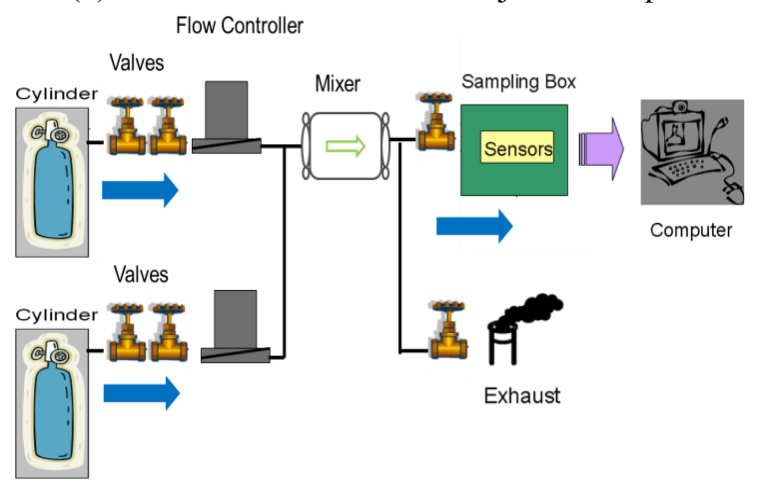

(b)When the measurements object is a gas

Fig. 3 Odor sensing system.

Here, seven sensors are used. Therefore, for one odor, there are seven sensor values, which will be used for classification. We have measured three types of odors. The sampling frequencies are $1[\mathrm{~Hz}]$. In Fig. 4, we show the measurement data for the sample where the horizontal axis is the measurement time and the vertical axis is the frequency shift deviation from the standard value $(20 \mathrm{M}[\mathrm{Hz}])$. They may include impulsive noises due to the typical phenomena of QCM sensors. To remove these impulsive noises we adopt a median filter which replaces a value at a specific time by a median value among neighboring data around the specific time.

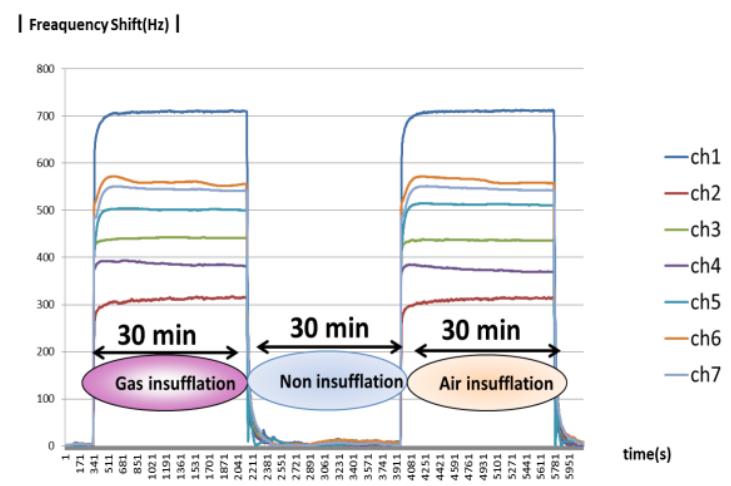

(a) Ethylene

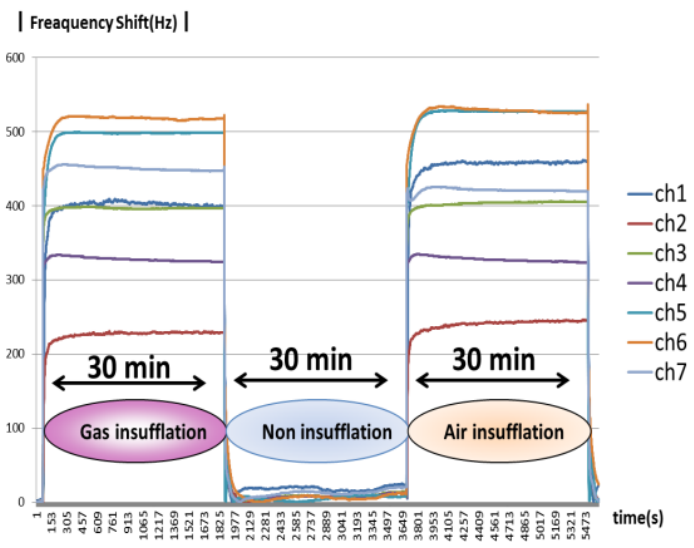

(b)Ethanol

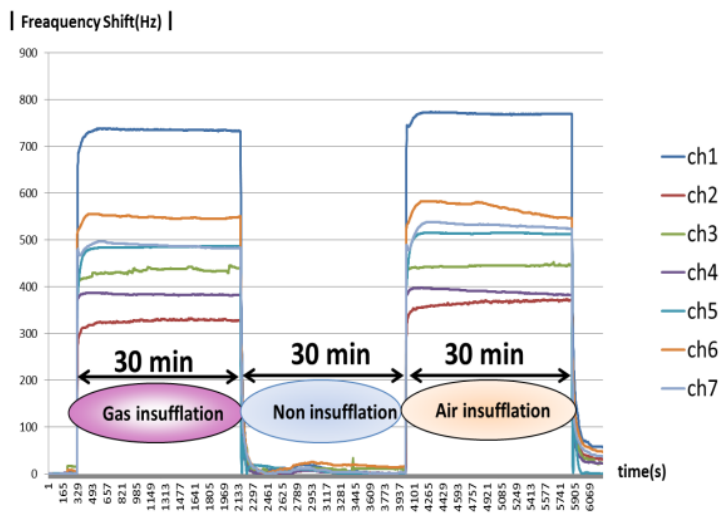

(c)Steam

Fig. 4 Sample of odor data.

\section{4 .1 Feature Extraction Methods}

We measured the data shown in Table 2. For each of the odor concentration, ethylene gas in only possible to measure $1 \mathrm{ppm}$ as a matter of convenience.

Table 2. Measured the data list

\begin{tabular}{|c|c|c|c|}
\hline Consentration(ppm) & Ethylene & Ethanol & Steam \\
\hline 1 & 3 data & & \\
\hline 10 & 1 data & 2 data & 2 data \\
\hline 100 & 1 data & 2 data & \\
\hline 110 & & & 2 data \\
\hline 1000 & 1 data & 2 data & 2data \\
\hline
\end{tabular}

We describe the feature extraction method in order to extract quantitative characteristics and suppress the variation of the data (Fig.5). First, it takes out the data of the injected gas from the measured data interval. We define $\operatorname{chi}=\{\mathrm{x} 0, \mathrm{x} 1, \ldots, \mathrm{xN}\}$, where $\mathrm{x} 0$ is a gas injection point, $x N$ is the measurement end point. Then, we derive a linear approximation curve from data. Let me be the $\mathrm{xJ}$ intersection of the data and the approximate curve. The time of the $\mathrm{xJ}$ data 
assuming the start time of the waveform, determine the average amount of change fave(chi) from xjby the following formula

$$
f_{\text {ave }}\left(\operatorname{ch}_{i}\right)=\frac{1}{N-j} \sum_{i=j}^{N}\left(x_{i}-x_{j}\right)
$$

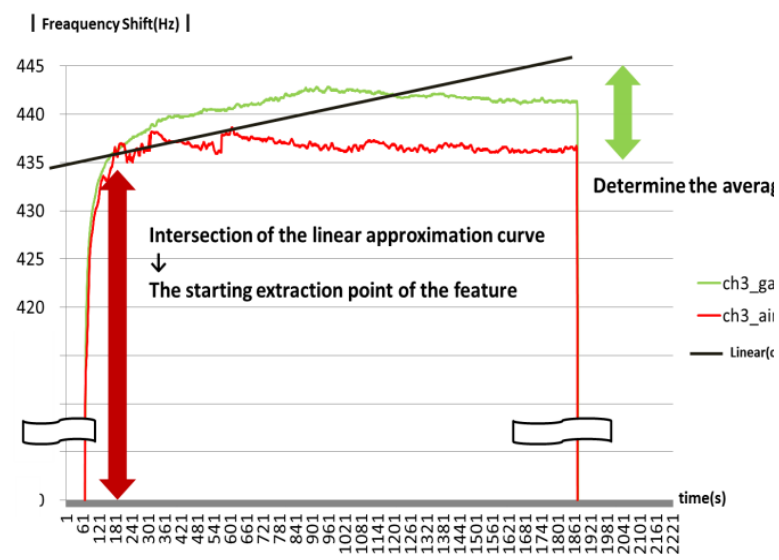

Fig. 5 Extract quantitative characteristic method.

In order to demonstrate the concentration dependence, we creating the graphs such as in Fig.6 using the sum of e feature vector obtained above. The vertical axis of each graph is the following formula

$$
\sum_{i=1}^{7} f_{\text {ave }}\left(c h_{i}\right)=\sum_{i=1}^{7} \frac{1}{N-j} \sum_{i=j}^{N}\left(x_{i}-x_{j}\right)
$$

Fig.6 (a) is a plot in the grid, Fig. 6(b) is obtained by logarithmic plot. Considering the results, QCM sensor was found to be proportional to the logarithm against the concentration. Hence, we determined a relational expression showing the concentration dependence in the logarithmic approximation. From these results, it was found that it is possible to estimate the concentration of the input data after odor classification.

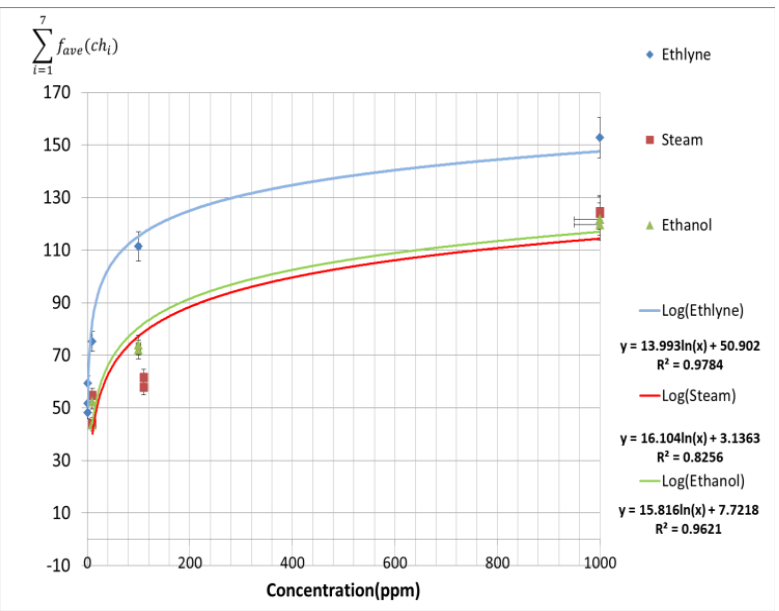

(a) Grid plot

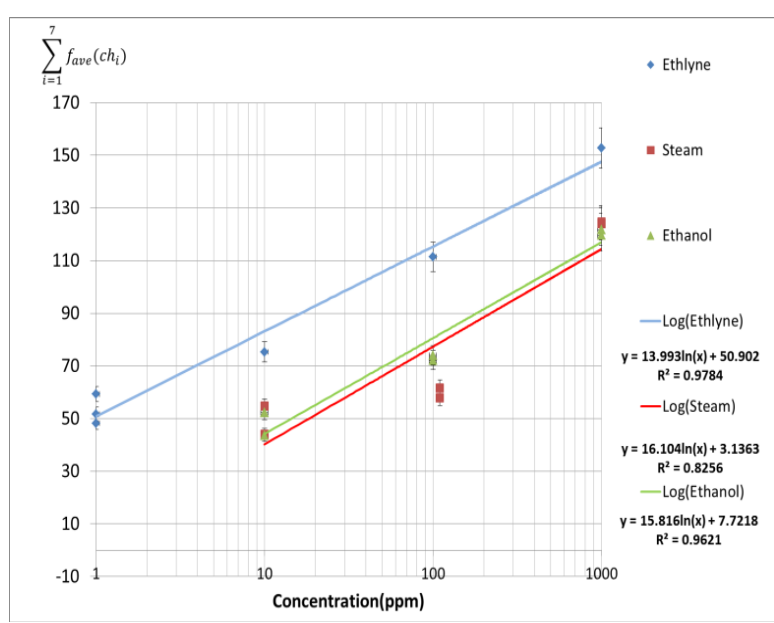

(b) Logarithmic plot

Fig. 6 Concentration dependence $\left(f_{\text {ave }}\left(c h_{i}\right)\right)$.

Further, frequency displacement of the QCM sensors considered the following equation is proportional to the amount of adsorption of odorants. $\Delta \mathrm{f} \propto \Delta \mathrm{m}=\mathrm{W}^{*} \mathrm{~V}^{*} \Delta \mathrm{C}$

Where, W: molecular weight of the odorant , V: Volume, C: concentration.

In this study, in order to normalize considering the concentration, the norm of the feature vector is Euclidean norm. Below we show the formula.

$$
\begin{gathered}
f_{\text {ave }_{\text {normal }}}\left(c h_{i}\right)=\frac{f_{i_{\text {ave }}}}{\|f\|} \\
|| f||=\sqrt{\sum_{i=1}^{s}\left(f_{\text {ave }}\left(c h_{i}\right)\right)^{2}}
\end{gathered}
$$

Then, we have created a graph with vertical axis the sum of the normalized feature vector in the same manner as above(Fig. 7). Therefore, it was confirmed that it is flat in relation to the concentration by the normalized considering the concentration.

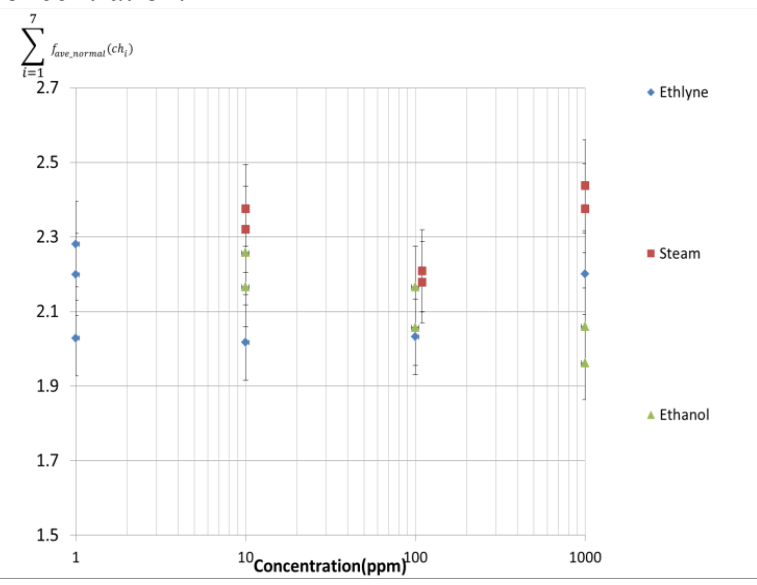

Fig .7 Concentration dependence $\left(f_{\text {ave }}\right.$ normal $\left.\left(c h_{i}\right)\right)$. 


\section{Classification Method of Odor Data}

We will show two types of neural networks: one is a multi-layered neural network based on error backpropagation method and the other is a learning vector quantization (LVQ).

First, we will explain the multi-layered neural networks. In order to classify the odors, we adopt a three-layered neural network based on the error back-propagation method, as shown in Fig.8. The error back-propagation algorithm, which is based on the gradient method, is given by the following steps.

Step 1 . Set the initial values of $w_{j i}, w_{k j}, \theta_{j}, \theta_{k}$, and $\eta(>0)$.

Step 2. Specify the desired values of the output $d_{k}, k=1,2, \cdots, K$ corresponding to the input data $\mathrm{x}_{\mathrm{i}}, \mathrm{i}=1,2, \ldots, \mathrm{I}$ in the input layer.

Step 3. Calculate the outputs of the neurons in the hidden layer by

$$
\begin{gathered}
n e t_{j}=\sum_{i=1}^{I} w_{j i} x_{i}-\theta_{j}, o_{j}=f\left(\text { net }_{j}\right), f(x) \\
=\frac{1}{1+e^{-x}}
\end{gathered}
$$

Step 4. Calculate the outputs of the neurons in the output layer by

$$
\begin{gathered}
\text { net }_{k}=\sum_{k=1}^{K} w_{k j i} O_{j}-\theta_{j}, O_{k}=f\left(\text { net }_{k}\right), f(x) \\
=\frac{1}{1+e^{-x}}
\end{gathered}
$$

Step 5. Calculate the error en and generalized errors

$$
\begin{gathered}
\text { by } e_{k}=d_{k}-O_{k} \\
\delta_{k}=\delta_{k} O_{k}\left(1-O_{k}\right) \\
\delta_{j}=\sum_{k=1}^{K} \delta_{k} w_{k j} O_{j}\left(1-O_{j}\right)
\end{gathered}
$$

Step 6. Use the following formula to calculate half of the sum of the squares of the errors in the output of all.

$$
\mathrm{E}=\frac{1}{2} \sum_{k=1}^{K} e_{k}{ }^{2}
$$

Step 7. If E is sufficiently small, exit the learning. Otherwise, modify the weight by the following equation:

$$
\begin{gathered}
\Delta w_{k j} \equiv w_{k j}(t+1)-w_{k j}(t)=\eta \delta_{j} O_{j k} \\
\Leftarrow w_{k j}+\Delta w_{k j}
\end{gathered}
$$

$$
\begin{gathered}
\Delta w_{j i} \equiv w_{j i}(t+1)-w_{j i}(t) \quad=\eta \delta_{i} O_{j} \\
\Leftarrow w_{j i}+\Delta w_{j i}
\end{gathered}
$$

Step 8. Go to Step 3.

Using the above recursive procedure, we can train the odor data.

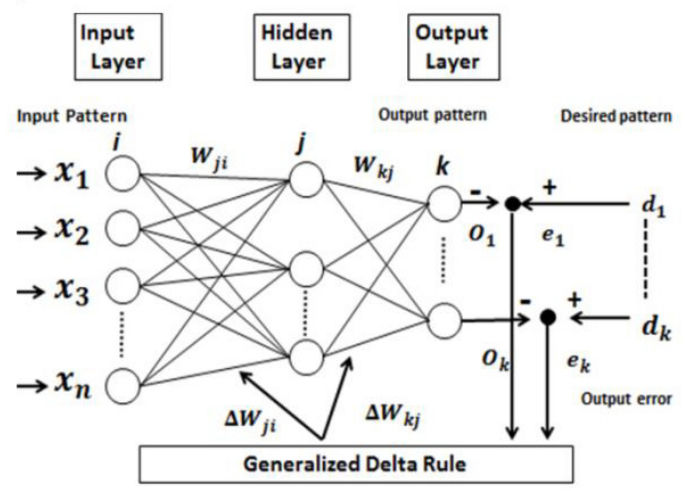

Fig. 8 Error-back propagation structure

Next, we will show the LVQ:The structure of LVQ is two layered, consisting of an input layer and a competitive layer as shown in Fig. 9.

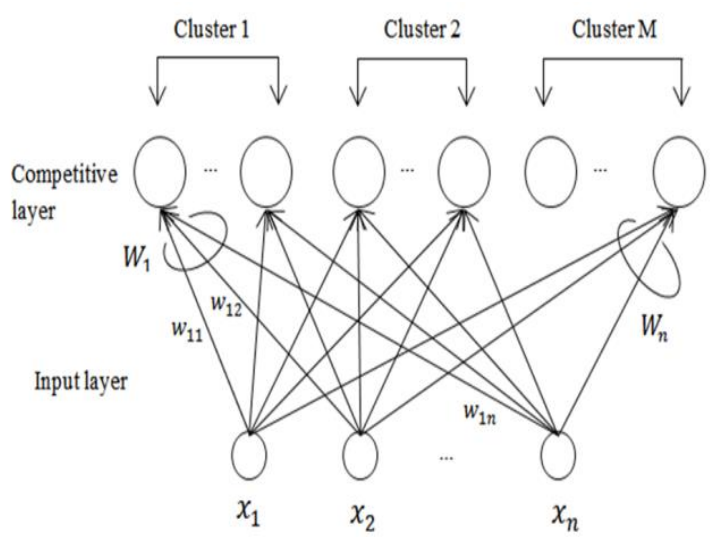

Fig. 9 LVQ structure

Learning vector quantization method as shown in Fig. 9 Learning vector quantization is a supervised learning method for the purpose of pattern classification for input data. The learning method is given by the following steps:

Step 1. Set the initial values of $w_{i j}(j=1,2, \ldots M$, $i=1,2, \ldots, n), T$, and $\alpha_{0}(>0)$ where $T$ is the total iteration number for learning, $n$ is the number of input, $M$ is the number of neurons in cluster $j$, and $\alpha_{0}$ is the initial value of the learning rate.

Step 2. First, calculate proximity to the coupling coefficient vector $\mathrm{WJ}$ of the input vector $\mathrm{x}$ and 
neuron $\mathrm{j}$ in the sense of Euclidean distance. The neuron with the closest coupling coefficient in the sense of Euclidean distance in the competitive layer neuron is detected by following equation for the input pattern.

$$
\begin{gathered}
d_{j}=\left\|\mathrm{x}-w_{j}\right\|=\sqrt{\sum_{i=1}^{n}\left(x_{i}-w_{j i}\right)^{2}} \\
d_{c}=\left\|\mathrm{x}-w_{c}\right\|=\min _{j} d_{j}
\end{gathered}
$$

Step 3. If the input vector and winning neuron c belong to the same class, then change $w_{j}(t)$ by using the following equation:

$$
\begin{array}{r}
w_{j}(t+1)=w_{j}(t)+\alpha(t)\left(x-w_{j}(t)\right), j=c \\
w_{j}(t+1)=w_{j}, j \neq c
\end{array}
$$

where

$$
\alpha(t)=\alpha_{0}\left(1-\frac{1}{T}\right) .
$$

If the input vector and neuron c belong to the different class, then change $w_{j}(t)$ by using the following equation:

$$
\begin{array}{r}
w_{j}(t+1)=w_{j}(t)-\alpha(t)\left(x-w_{j}(t)\right), j=c \\
w_{j}(t+1)=w_{j}, j \neq c .
\end{array}
$$

Step 4. If $\mathrm{t}<\mathrm{T}$, Go to Step 2

Using the above recursive procedure, we can train the odor data.

\section{Results}

In order to classify the feature vector by using the error-back propagation, we allocate the desired output for the input feature vector, which is a sevendimensional vector, as shown in Table 3. By adding the coefficient of variation to the usual feature vector, the variations for odors are reduced. The training was performed until the total error was less than or equal to $1 \times 10-2$ where $\eta=0$.

We have examined two algorithms of a learning vector quantization and error back propagation. In learning vector quantization and error back propagation, the training sample number $\mathrm{P}^{\prime}=6$ and test sample number is three.

The total number of classification of 40 test samples is checked. The results are summarized in Table 4 and Table 5.

Table 3. Training data set

\begin{tabular}{|c|c|c|c|}
\hline Symbols & Output A & Output B & Output C \\
\hline A & 1 & 0 & 0 \\
\hline B & 0 & 1 & 0 \\
\hline C & 0 & 0 & 1 \\
\hline
\end{tabular}

Table 4. Classification results for learning vector quantization

\begin{tabular}{|ccc|c|c|}
\hline \multirow{2}{*}{ Odor data } & \multicolumn{4}{c|}{ Classification results (Average= $\mathbf{7 0} \%$ ) } \\
\cline { 2 - 5 } & Ethylene & Ethanol & Steam & Correct \\
\hline Ethylene & 86 & 2 & 32 & $72 \%$ \\
\hline Ethanol & 0 & 110 & 10 & $92 \%$ \\
\hline Steam & 29 & 35 & 56 & $47 \%$ \\
\hline
\end{tabular}

Table 5. Classification results for layered neural networks

\begin{tabular}{|cc|c|c|c|}
\hline \multirow{2}{*}{ Odor data } & \multicolumn{4}{c|}{ Classification results (Average=78\%) } \\
\cline { 2 - 5 } & Ethylene & Ethanol & Steam & Correct \\
\hline Ethylene & 99 & 0 & 21 & $83 \%$ \\
\hline Ethanol & 0 & 108 & 12 & $90 \%$ \\
\hline Steam & 27 & 18 & 75 & $63 \%$ \\
\hline
\end{tabular}

We have presented the reliability of a new system designed from various kinds of QCM sensors. We have shown that after training the neural network for each odor, we were able to classify the various concentration odors.

In this paper, layered neural networks method became good results better used in the ethylene gas detection system.

\section{Conclusions}

As a result of principal component analysis using feature vectors data, ethanol data are separated as shown in Fig. 10.From these results, it was found that it can be classified by using the NN. Nevertheless, it seems to be difficult to determine the decision boundary regarding steam and ethanol. Therefore, it is necessary to concerned about concentration dependence of samples by concentration range further to make algorithms to estimate the concentration of data classified important challenges and efforts. In particular we believe that it is necessary to extract a feature vector that is independent of the concentration. Moreover, we need to build an algorithm that allows the classification and concentration estimation from the mixed odor. 


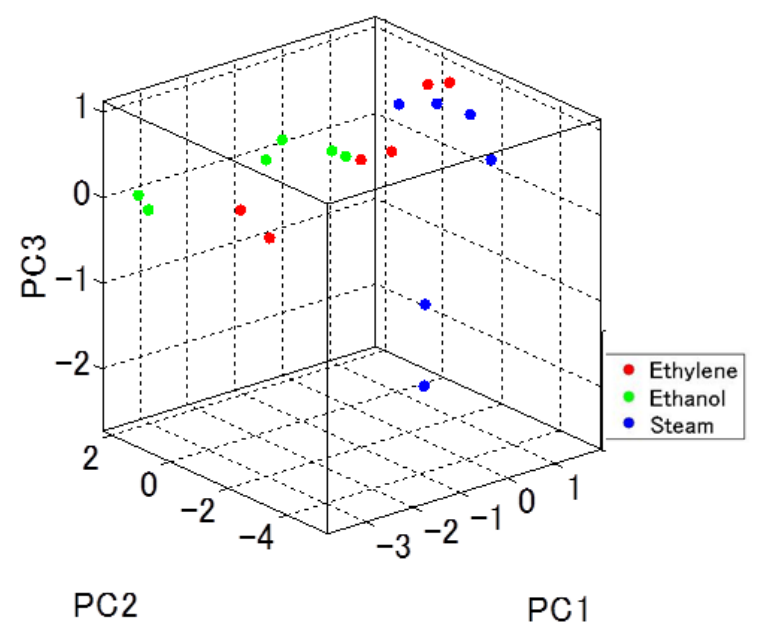

(a) Original feature vector data

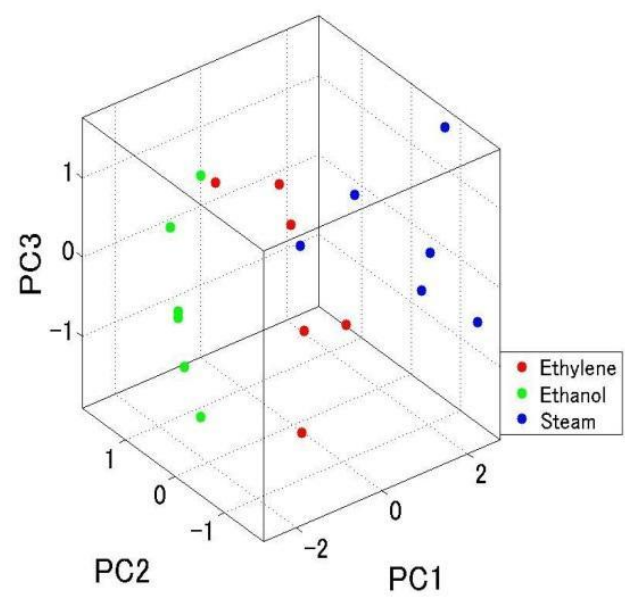

(b) Normalization feature vector data

Fig10. Principal Component Analysis

\section{Acknowledgement}

This work was supported by JSPS KAKENHI Grant-in-Aid for challenging Exploratory Research (25630180). The authors would like to thank JSPS to support this research work.

\section{References}

[1] S.Omatu, Processing of odor information, Journal of Signal Processing, Vol. 14, No. 3 pp.167-175, May(2010)

[2] S. Omatu, Processing of odor information, Journal of Signal Processing, Vol.14, No.5 pp .329-338, September (2010)

[3] S. Omatu,T. Wada,S. Roriguez,P. Chamos,J. Corchado, Multi-agent Technology to Perform Odor Classification, Advances in Intelligent Systems and Computing, Spain ,pp 241-252, (2014)

[4] H. Moriizumi, T. Nakamoto, Sensor Engineering, ;SHOKODO Co.,Ltd. (1997)

[5] D Chamovitz, Do Plants Think?, Scientific American, (2010).

[6] S. Omatu and M. Yano, Intelligent Electronic Nose System Independent on Odor Concentration, International Symposium on Distributed Computing and Artificial Intelligence,Salamanca, Spain, pp. 1-9. (2011)

[7] T. Kohonen, Self-Organizing Maps, Springer (1997) 